\title{
Sanguinarine protects against ovariectomy-induced osteoporosis in mice
}

\author{
YAN MA $^{1 *}$, JUNJIE CHU ${ }^{2 *}$, JIANJUN MA ${ }^{1}$, LEI NING $^{1}$, KE ZHOU $^{1}$ and XIANGQIAN FANG ${ }^{1}$ \\ Departments of ${ }^{1}$ Orthopedic Surgery and ${ }^{2} \mathrm{Head}$ and Neck Surgery, Sir Run Run Shaw Institute of Clinical Medicine, \\ Sir Run Run Shaw Hospital, Medical College of Zhejiang University, Hangzhou, Zhejiang 310016, P.R. China
}

Received March 29, 2016; Accepted March 14, 2017

DOI: $10.3892 / \mathrm{mmr} .2017 .6574$

\begin{abstract}
Natural compounds are alternative agents that have therapeutic potential for preventing and treating osteoporosis. Traditionally, sanguinarine has been used clinically due to its diverse biological properties, including antimicrobial, anti-inflammatory and anticancer effects. Recently, for the first time, it was reported that sanguinarine inhibits osteoclast differentiation and bone resorption by suppressing the tumor necrosis factor ligand superfamily member 11-induced nuclear factor- $\kappa \mathrm{B}$ and extracellular signal-regulated kinase signaling pathways in vitro. Therefore, the present study further investigated the pharmacological effect of sanguinarine on osteoporosis in vivo. Micro-computed tomography and histomorphometry analysis demonstrated that sanguinarine, at low and high concentrations, prevents ovariectomy (OVX)-induced bone loss. In addition, further investigation of the cellular response in vivo revealed that sanguinarine inhibited osteoclastic bone resorption and promoted osteoblastic bone formation in a dose-dependent manner. Therefore, the present study demonstrated that sanguinarine protected mice from OVX-induced osteoporosis by modulating bone remodeling, indicating that sanguinarine may have potential in the treatment of osteoporosis.
\end{abstract}

\section{Introduction}

Osteoporosis is a medical and socioeconomic problem characterized by the loss of bone mass and mechanical properties, leading to an increased risk of fracture. A variety of factors may lead to osteoporosis, and menopause is one of the most common reasons (1). In postmenopausal women, decreased

Correspondence to: Dr Xiangqian Fang, Department of Orthopedic Surgery, Sir Run Run Shaw Institute of Clinical Medicine, Sir Run Run Shaw Hospital, Medical College of Zhejiang University, 3 East Qingchun Road, Hangzhou, Zhejiang 310016, P.R. China

E-mail: orthopaedics2009@hotmail.com

${ }^{*}$ Contributed equally

Key words: sanguinarine, osteoporosis, bone remodeling estrogen levels cause increased osteoclast formation and elevated bone resorption, resulting in more rapid bone loss (2). Therefore, targeting osteoclast formation and function is one of the strategies for preventing and treating osteoporosis. Currently, several drugs aimed at inhibiting osteoclast formation or function have been used for osteoporosis, including bisphosphonates and denosumab (3). Aside from these synthesized compounds or antibodies, natural compounds are alternative therapeutic agents for the prevention and treatment of osteoporosis. Several natural products have exhibited an anti-osteoporotic property, including berberine, kaempferol, formononetin and osthole (4-7). Our study group has a long-term interest in evaluating the pharmacological effect of natural compounds on osteoporosis (8-11). Sanguinarine [13-methyl-(1,3) benzodioxole (5,6-c)-1,3-dioxolane (4,5-I) phenanthridinium] is an alkaloid derived from the roots of Sanguinaria canadensis. Sanguinarine exhibits multiple pharmacological effects, including anti-inflammatory, antitumor, antimicrobial, antiplatelet and antihypertensive properties (12). The authors previously revealed that sanguinarine inhibited osteoclast formation and bone resorption by suppressing the tumor necrosis factor ligand superfamily member 11-induced nuclear factor- $\kappa \mathrm{B}$ and extracellular signal-regulated kinase signaling pathways (10). However, whether this natural compound prevents estrogen deficiency-induced bone loss requires further investigation in vivo. Considering the potential of sanguinarine for suppressing osteoclast formation in vitro, and the wide use of this compound in traditional medicine, the present study was designed to investigate whether sanguinarine may protect against ovariectomy (OVX)-induced osteoporosis and, thus, be a potential agent for future clinical application.

\section{Materials and methods}

Ethics statement. The Animal Care and Experiment Committee of Zhejiang University School of Medicine (Zhejiang, China) approved all experimental procedures and the study was performed according to the guidelines for Ethical Conduct in the Care and Use of Nonhuman Animals in Research by the American Psychological Association (13).

Media and reagents. Sanguinarine, dimethyl sulfoxide (DMSO) and the tartrate-resistant acid phosphatase (TRAP) staining kit were purchased from Sigma-Aldrich (Merck 
KGaA, Darmstadt, Germany). Sanguinarine was dissolved in DMSO and was stored at $-20^{\circ} \mathrm{C}$. To prevent photosensitivity, the experiments were performed in the absence of visible light. Sanguinarine was diluted using PBS so that DMSO comprised $<0.1 \%$ of the total volume, to a final concentration of $2 \mathrm{mg} / \mathrm{ml}$.

Animals and study design. An OVX-induced osteoporosis mouse model was established to determine the effects of sanguinarine on osteoporosis in vivo. Healthy 8-week-old female C57BL/6J mice ( $\mathrm{n}=28$; weight, $19.11 \pm 0.57 \mathrm{~g}$ ) received either a sham operation $(n=7)$ or OVX $(n=21)$ under anesthesia using $8 \%$ chloral hydrate. Mice were housed in an animal facility under temperature $\left(22-24^{\circ} \mathrm{C}\right)$ and humidity $(50-60 \%)$ controlled conditions, with a $12 \mathrm{~h}$ light/dark cycle and with free access to food and water. The ovary was excised in OVX-operated mice, and the adipose tissue around the ovary was excised in sham-operated mice, as described previously (14). Mice were assigned to the following four groups: Sham with PBS control (sham; n=7; weight, 18.57 $\pm 0.67 \mathrm{~g}$ ), OVX with PBS (vehicle; $\mathrm{n}=7$; weight, $19.07 \pm 0.45 \mathrm{~g}$ ), OVX with low sanguinarine ( $5 \mathrm{mg} / \mathrm{kg} ; \mathrm{n}=7$; weight, $19.43 \pm 0.61 \mathrm{~g})$, OVX with high sanguinarine $(10 \mathrm{mg} / \mathrm{kg} ; \mathrm{n}=7$; weight, $19.36 \pm 0.57 \mathrm{~g})$. At 1 week post-operation, all mice were intraperitoneally injected with PBS or sanguinarine twice a week for 6 weeks. At the end of experiment, the mice were sacrificed. The left legs were excised and fixed in $4 \%$ paraformaldehyde for $48 \mathrm{~h}$ under controlled temperature $\left(22-24^{\circ} \mathrm{C}\right)$, for micro-computed tomography (CT) analysis. The right legs were immediately removed and snap-frozen in liquid nitrogen for RNA isolation. The uteruses were excised and weighed. In the sham group the uterine weight was $82.60 \pm 15.30 \mathrm{mg}$, in the OVX groups the uterine weights were $15.86 \pm 1.36,13.67 \pm 2.23$ and $11.5 \pm 2.68 \mathrm{mg}$, in the vehicle, low sanguinarine and high sanguinarine groups, respectively.

RNA isolation and reverse transcription-quantitative polymerase chain reaction $(R T-q P C R)$ analysis. RT-qPCR was performed to determine the expression of genes associated with bone metabolism. Total RNA from the tissues was isolated using TRIzol ${ }^{\circledR}$ reagent (Invitrogen; Thermo Fisher Scientific, Inc., Waltham, MA, USA) and RT was performed using $1.0 \mu \mathrm{g}$ total RNA and the HiFiScript cDNA kit (CWBIO, Beijing, China), according to the manufacturer's protocol. Amplification reactions were set up in $20 \mu 1$ reaction volumes containing amplification primers and UltraSYBR Mixture (with ROX; CWBIO Co., Ltd.), expression was detected by the ABI 7500 Sequencing Detection System (Applied Biosystems; Thermo Fisher Scientific, Inc.). cDNA (1 $\mu \mathrm{l})$ was used in each amplification reaction. The thermocycling conditions of the reaction were as follows: Activation at $95^{\circ} \mathrm{C}$ for $10 \mathrm{~min}$; 40 cycles of amplification, $95^{\circ} \mathrm{C}$ for $10 \mathrm{sec}, 60^{\circ} \mathrm{C}$ for $24 \mathrm{sec}$ and $72^{\circ} \mathrm{C}$ for $20 \mathrm{sec}$; final extension at $72^{\circ} \mathrm{C}$ for $1 \mathrm{~min}$. All the reactions were performed in triplicate and were normalized to the housekeeping genes 18S ribosomal RNA (18S) and GAPDH. Expression was quantified using the $\Delta \Delta \mathrm{Cq}$ method, as described previously (15). The primer sequences were as follows: $18 \mathrm{~S}$, 5'-CCTGCGGCTTAATTTGACTC-3' (forward) and 5'-AAC TAAGAACGGCCATGCAC-3' (reverse); GAPDH, 5'-ACC CAGAAGACTGTGGATGG-3' (forward) and 5'-CACATT
GGGGGTAGGAACAC-3' (reverse); TRAP, 5'-CCATTGTTA GCCACATACGG-3' (forward) and 5'-CACTCAGCACAT AGCCCACA-3' (reverse); T-cell immune regulator 1 (Tcirg1), 5'-TGGCTACCGTTCCTATCCTG-3' (forward) and 5'-CTT GTCCGTGTCCTCATCC-3' (reverse); cathepsin K (CtsK), 5'-TCCGCAATCCTTACCGAATA-3' (forward) and 5'-AAC TTGAACACCCACATCCTG-3' (reverse); nuclear factor of activated T-cells 1 (NFATc1), 5'-TCCACCCACTTCTGACTT CC-3' (forward) and 5'-CTTCGCCCACTGATACGAG-3' (reverse); alkaline phosphatase (ALP), 5'-ACTGGCTGTGCT CTCCCTAC-3' (forward) and 5'-GAAGTTGCCTGGACC TCTCC-3' (reverse); osteopontin (OPN), 5'-TGATGATGA CGATGGAGACC-3' (forward) and 5'-GGGACGATTGGA GTGAAAGT-3' (reverse); runt related transcription factor 2 (Runx2), 5'-CCTCTGACTTCTGCCTCTGG-3' (forward) and 5'-ATGAAATGCTTGGGAACTGC-3' (reverse); ColI 5'-CAT CGTGGCTTCTCTGGTCT-3' (forward) and 5'-CCGTTG AGTCCGTCTTTGC-3' (reverse); dentin matrix acidic phosphoprotein 1 (Dmp1), 5'-GCTACATTGCTTTGGCTCCT-3' (forward) and 5'-GGTCACTTCCTGTCCTGCTC-3' (reverse); phosphate regulating endopeptidase homolog X-linked (Phex), 5'-CCGAACCAGTGAGGCTATGT-3' (forward) and 5'-CGA GGGACCAATGTCTTTCA-3' (reverse); and sclerostin (SOST), 5'-CGTGCCTCATCTGCCTACTT-3' (forward) and 5'-AGGTCTGCCTCCATTCTCC-3' (reverse).

Micro-CT scanning. The left tibia of each mouse was fixed with $4 \%$ paraformaldehyde for $48 \mathrm{~h}$ under controlled temperature $\left(22-24^{\circ} \mathrm{C}\right)$ and washed with PBS three times, it was subsequently stored in $70 \%$ ethanol until $\mathrm{CT}$ scanning. The fixed tissues were analyzed using a high-resolution micro-CT (SkyScan 1072; Bruker microCT; Bruker Corporation, Billerica, MA, USA). The scanning protocol was set at an isometric resolution of $9 \mu \mathrm{m}$, and the radiography energy settings were $80 \mathrm{kV}$ and $80 \mu \mathrm{A}$. Following reconstruction, the region of interest for the tibia was set at $0.5 \mathrm{~mm}$ from the proximal femoral growth plate and was selected for further qualitative and quantitative analysis. Several structural parameters, including the trabecular bone volume/total volume (BV/TV), mean trabecular thickness (Tb.Th), mean trabecular number (Tb.N) and mean trabecular separation (Tb.Sp) were measured by micro-CT as reported previously (16).

Histological analysis. The left femur of each mouse was fixed in $4 \%$ paraformaldehyde for $48 \mathrm{~h}$ under controlled temperature $\left(22-24^{\circ} \mathrm{C}\right)$ and decalcified in $12 \%$ EDTA. Decalcified tissues were paraffin-embedded and sectioned (thickness, $8 \mu \mathrm{m})$. For histological examination, sections were stained with hematoxylin for $5 \mathrm{~min}$ and with eosin for $2 \mathrm{~min}$ under controlled temperature $\left(22-24^{\circ} \mathrm{C}\right)$, and another section was stained with the TRAP staining kit to identify osteoclasts on the bone surface. Stained sections were imaged using a photomicroscope (magnification, x100-200), and the number of osteoblasts/bone perimeter (N.Ob/B.Pm), empty lacuna rate, percentage of osteoclast surface/bone surface(Ocs/BS), number of osteoclasts/field of tissue and microstructure parameters, including BV/TV, Tb.Th, Tb.N and Tb.Sp, were measured and quantified using Image Pro-Plus software (version 4.0; Media Cybernetics, Inc., Rockville, MD, USA) (11). 
A

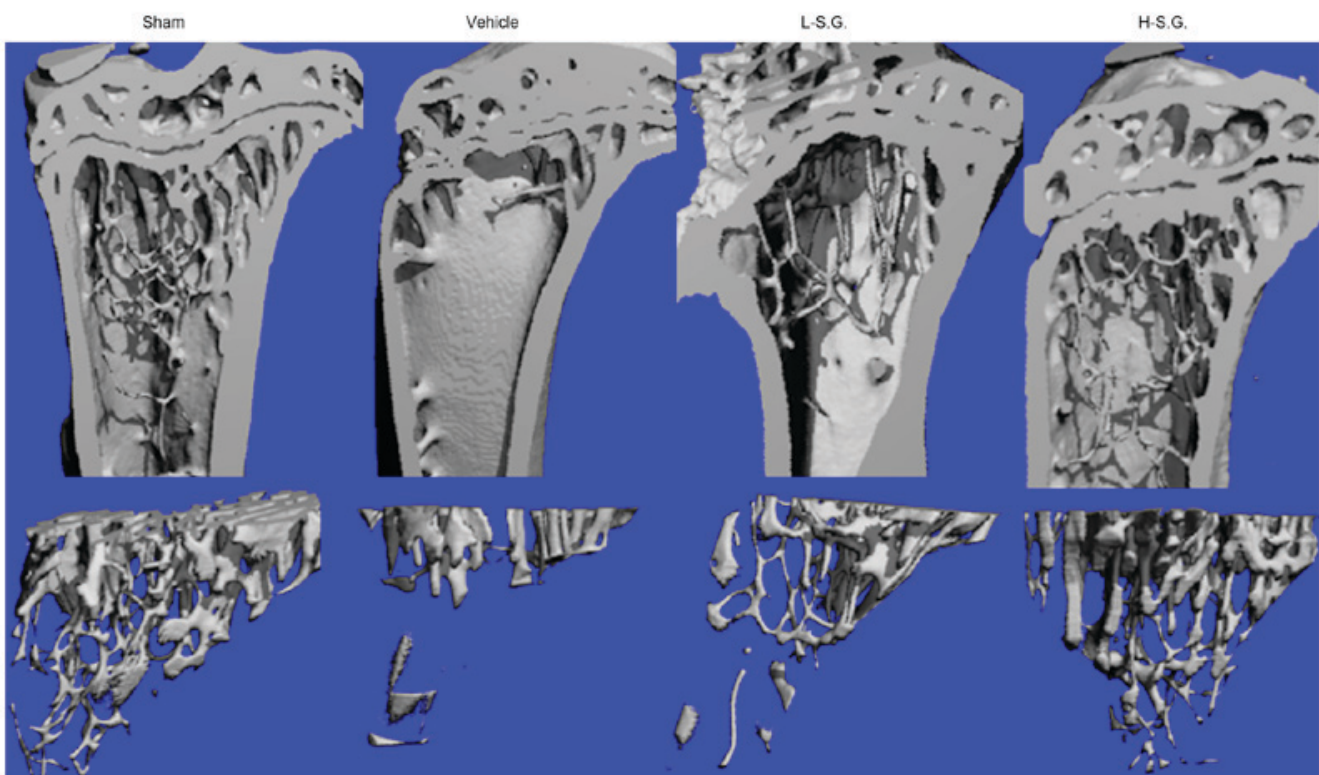

B

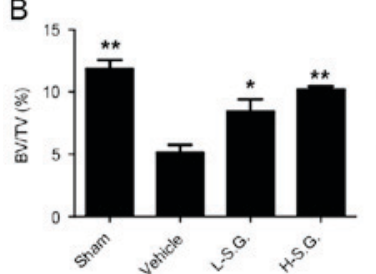

C

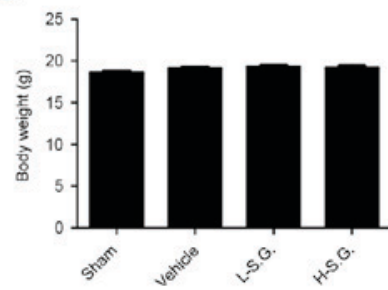

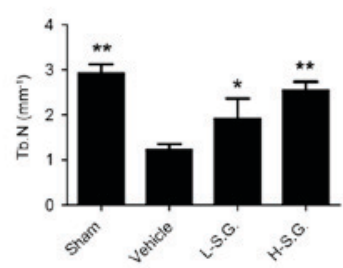

D

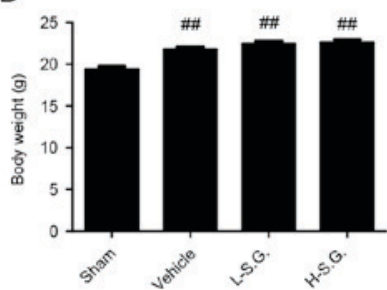

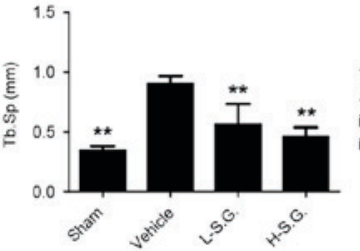

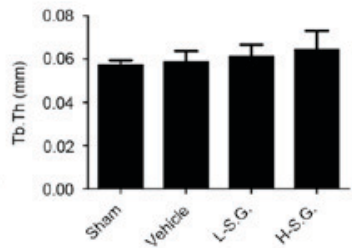

E

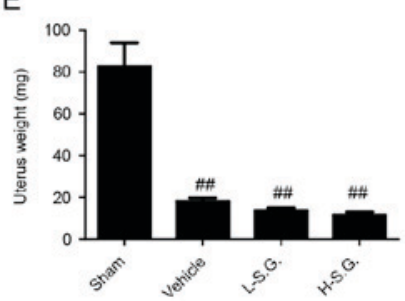

Figure 1. Sanguinarine prevents ovariectomy-induced bone loss in vivo. (A) Fixed tibiae were analyzed by micro-computed tomography and three-dimensional reconstructed images from each group are presented. (B) BV/TV, Tb.N, Tb.Sp and Tb.Th were caculated for sham, vehicle, L-S.G. and H-S.G. groups. (C) Initial (8-weeks-old) and (D) final (15-weeks-old) body weights of mice. (E) Uteri were dissected and weighed at the end of the study ( 15 -weeks-old). "P<0.05 and ${ }^{* *} \mathrm{P}<0.01$ vs. vehicle; ${ }^{\# \#} \mathrm{P}<0.01$ vs. sham. BV/TV, trabecular bone volume/total volume; Tb.N, trabecular number; Tb.Sp, mean trabecular separation; Tb.Th, mean trabecular thickness; L-S.G., low-dose sanguinarine group; H-S.G., high-dose sanguinarine group.

Statistical analysis. GraphPad Prism 6.0 (GraphPad Software, Inc., La Jolla, CA, USA) was used to perform all statistical analyses. Data are presented as the mean \pm standard error of the mean. Statistical significance was determined by one-way analysis of variance, followed by the Dunnett's post hoc test to make comparisons between the groups. $\mathrm{P}<0.05$ was considered to indicate a statistically significant difference.

\section{Results}

Sanguinarine prevents $O V X$-induced bone loss in vivo. Micro-CT was used to determine the microarchitectural changes of the tibiae (Fig. 1A). The BV of the OVX vehicle group was significantly reduced compared with the sham group (BV/TV, $11.85 \pm 0.42$ and $5.14 \pm 0.36 \%$, respectively; Fig. 1B). Following treatment with sanguinarine, a protective effect against OVX-induced bone loss was observed in a dose-dependent manner. The BV/TV in the low-dose group was $8.42 \pm 0.56 \%$, which was $\sim 30 \%$ higher compared with the OVX vehicle group. In addition, the BV/TV in the high-dose group was increased by $50 \%$ compared with the OVX vehicle group. In addition, changes in the other bone mineral density indices (Tb.Th, Tb.N, Tb.Sp) occurred accordingly. The administration of sanguinarine significantly increased BV/TV and $\mathrm{Tb}$. N, and significantly reduced $\mathrm{Tb}$. Sp compared with the OVX vehicle group. Furthermore, the four groups of mice had a similar mean body weight prior to OVX (Fig. 1C). Despite the same feeding regimen, the final body weight of mice in the OVX groups was significantly increased compared with the sham group (Fig. 1D), while the uterine weight of mice in the OVX groups was significantly lower compared with the sham group when sacrificed (Fig. 1E). The results for body and uterine weight upon sacrifice indicate that the OVX model was established successfully. However, no difference was observed between the OVX vehicle group and OVX groups treated with high or low-dose sanguinarine, indicating that sanguinarine was not able to prevent OVX-induced weight 
A

A

Vehicle

L-S.G.

H-S.G.

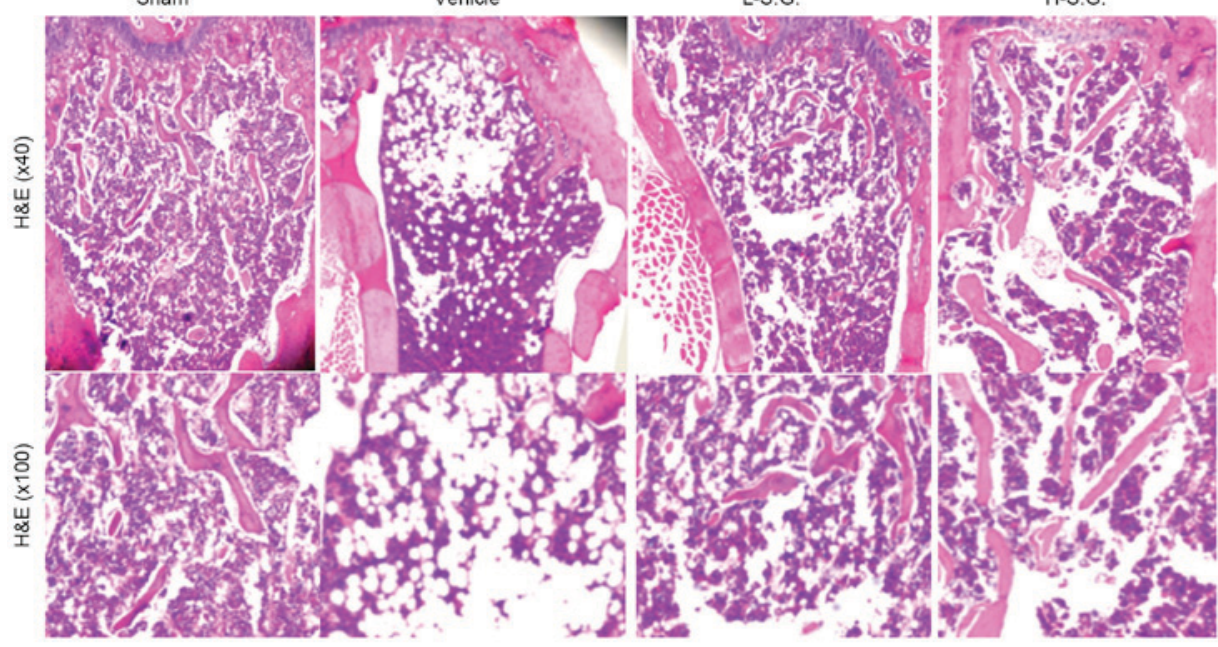

B
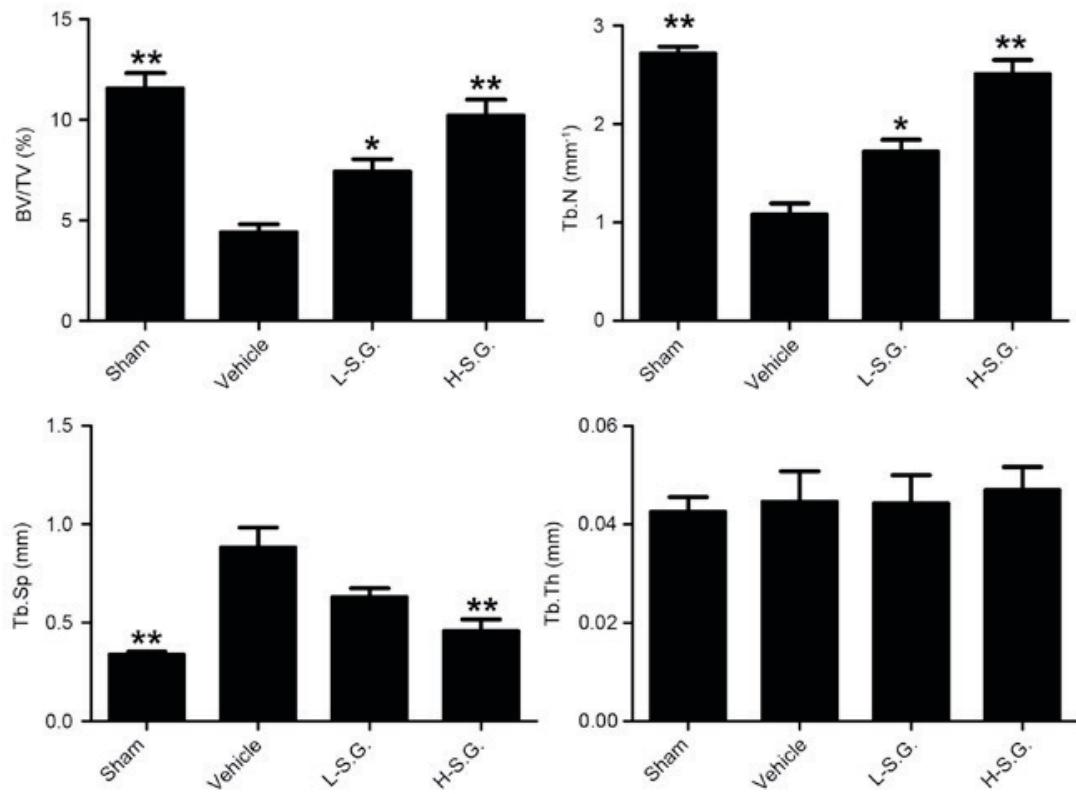

Figure 2. Histological analysis demonstrating that sanguinarine protects mice from ovariectomy-induced bone loss in vivo. (A) Decalcified tissues were paraffin-embedded and sectioned for H\&E staining. (B) BV/TV, Tb.N, Tb.Sp and Tb.Th were calculated for sham, vehicle, L-S.G. and H-S.G. groups. "P<0.05 and ${ }^{* *} \mathrm{P}<0.01$ vs. vehicle. H\&E, hematoxylin and eosin; BV/TV, trabecular bone volume/total volume; Tb.N, mean trabecular number; Tb.Sp, mean trabecular separation; Tb.Th, mean trabecular thickness; L-S.G., low-dose sanguinarine group; H-S.G., high-dose sanguinarine group.

gain and uterine atrophy. Collectively, the results indicated a protective effect of sanguinarine against OVX-induced bone loss in vivo.

Histological analysis indicates that sanguinarine protects mice from $O V X$-induced bone loss in vivo. Decalcified bone tissue was analyzed by H\&E staining (Fig. 2A). Consistent with the micro-CT results, the vehicle group exhibited a significantly decreased BV compared with the sham group (BV/TV, $4.40 \pm 0.24$ and $11.56 \pm 0.44 \%$, respectively; Fig. 2B). By contrast, sanguinarine at low and high concentrations prevented OVX-induced bone loss to a certain extent (BV/TV, 7.42 \pm 0.36 and $10.21 \pm 0.45 \%$ in the low- and high-dose groups, respectively). In addition, OVX vehicle mice also exhibited a significantly reduced Tb.N and significantly increased Tb.Sp compared with the sham group, while sanguinarine dose-dependently reversed this trend by increasing the Tb.N and reducing the Tb.Sp compared with the OVX vehicle group (Fig. 2B).
Sanguinarine inhibits osteoclastic bone resorption in vivo. TRAP staining was performed to identify osteoclasts on the bone surface (Fig. 3A), which revealed a reduced osteoclast number following sanguinarine treatment, with $53.63 \pm 3.02$ osteoclasts in the OVX vehicle group and 23.34 \pm 2.55 osteoclasts in the sanguinarine high-dose treatment group. The resorptive area, determined by the Ocs/BS, also supported the inhibitory effect of sanguinarine on osteoclastic bone resorption in vivo, with a $10.78 \pm 0.82 \%$ resorptive area in the OVX vehicle group and a $4.04 \pm 0.38 \%$ resorptive area in the sanguinarine high-dose treatment group (Fig. 3B). In addition, the suppressive effect of sanguinarine on osteoclasts was further supported by the osteoclast-specific gene expression profile. qPCR analysis demonstrated that osteoclastic markers, including TRAP, NFATc1, Tcirg1 and CtsK, were significantly upregulated in OVX vehicle mice compared with the sham group (Fig. 3C). The administration of sanguinarine treatment dose-dependently reduced the upregulation of osteoclast 

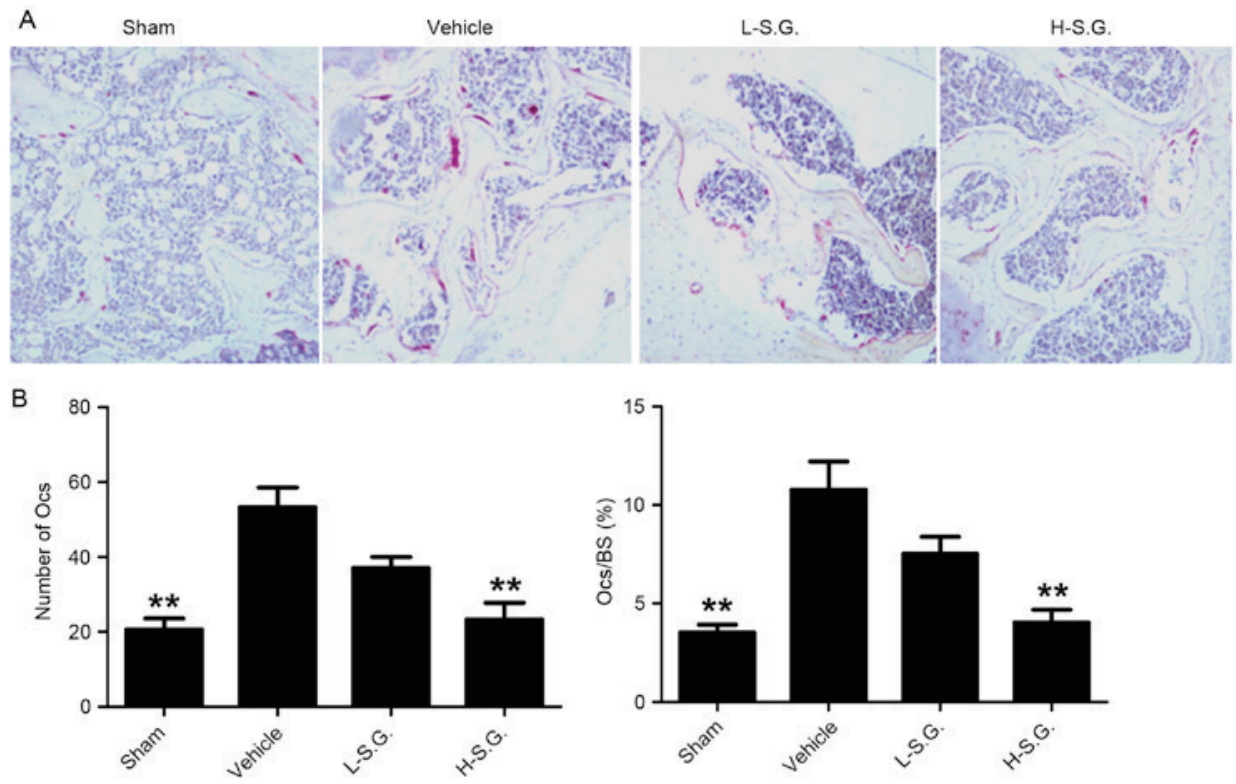

C
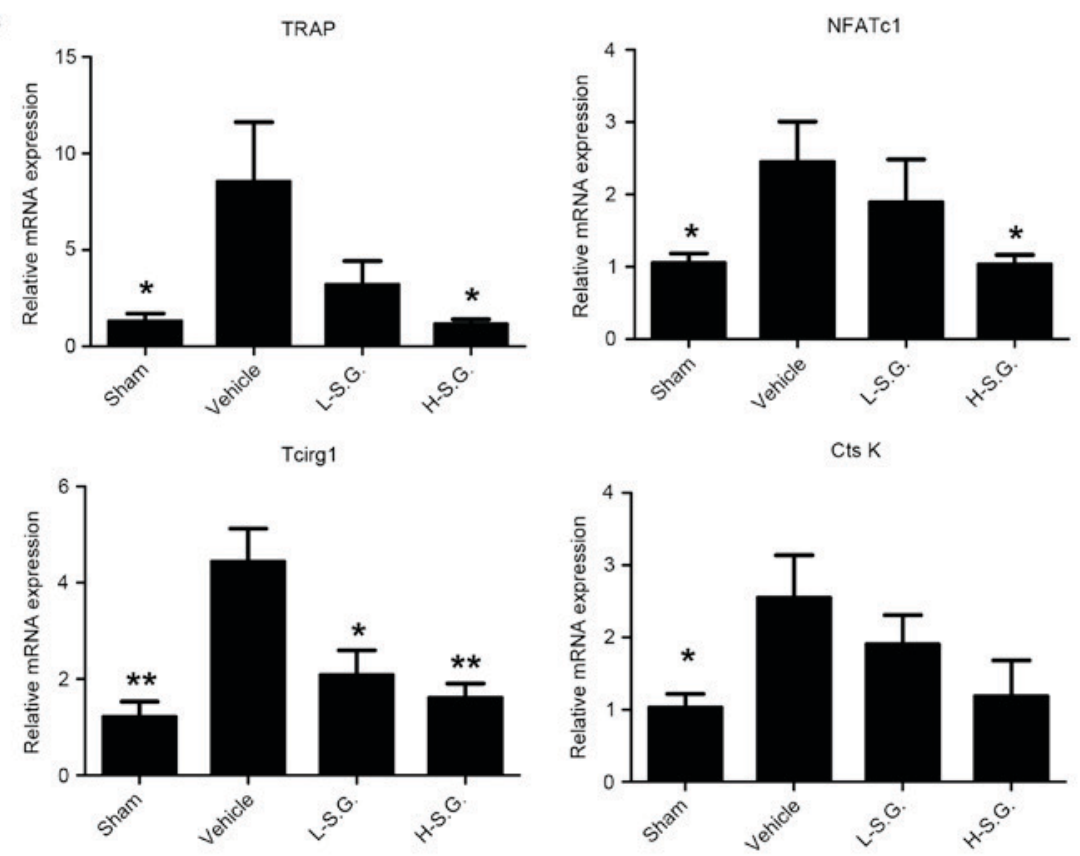

Figure 3. Sanguinarine inhibits osteoclastic bone resorption in vivo. (A) Decalcified tissues were paraffin-embedded and sectioned for TRAP staining. (B) Number of Ocs/field of tissue and the Ocs/BS were analyzed. (C) Expression of Oc-specific genes TRAP, NFATc1, Tcirg1 and CtsK was detected by quantitative polymerase chain reaction, and the results were normalized to the expression of 18S and GAPDH. Magnification, 100x. Yellow arrows, osteoblast; green arrows, Oc lacunae; black arrows, empty lacunae. * $\mathrm{P}<0.05$ and ${ }^{* *} \mathrm{P}<0.01$ vs. vehicle. TRAP, tartrate-resistant acid phosphatase; Ocs, osteoclasts; Ocs/BS, percentage of Oc surface/bone surface; NFATc1, nuclear factor of activated T-cells 1; Tcirg1, T-cell immune regulator 1; CtsK, cathepsin K; 18S, 18S ribosomal RNA; L-S.G., low-dose sanguinarine group; H-S.G., high-dose sanguinarine group.

activity markers in the bone tissue. Therefore, the results indicate that sanguinarine may protect against OVX-induced osteoporosis by inhibiting osteoclastic formation and bone resorption in vivo.

Sanguinarine promoted osteoblastic bone formation in vivo. Following H\&E staining (Fig. 4A), the N.Ob/B.Pm was calculated to determine the effect of sanguinarine on osteoblastic bone formation in vivo (Fig. 4B). The results demonstrated that the administration of sanguinarine dose-dependently increased the osteoblast number compared with OVX vehicle mice (Fig. 4B). The osteocyte number, osteocyte lacunae number and empty lacunae were also observed, and the empty lacunae rate was calculated to determine the effect of sanguinarine on osteocytes (Fig. 4C). The empty lacuna rate was increased in the vehicle group compared with the sham group $(44.40 \pm 2.66$ and $13.47 \pm 1.88 \%$, respectively). In addition, lowand high-dose sanguinarine reduced the empty lacuna rate to $26.66 \pm 1.02$ and $12.76 \pm 0.75 \%$ in the vehicle and sham groups, respectively. qPCR analysis further supported the results, which demonstrated that sanguinarine treatment increased the expression of osteoblast-specific genes, including Runx2, ALP, OPN and ColI compared with OVX vehicle mice (Fig. 4D). Furthermore, sanguinarine also increased the expression of osteocyte-specific genes, including Dmp1, Phex and SOST in vivo compared with the OVX vehicle group (Fig. 4E). The 
A
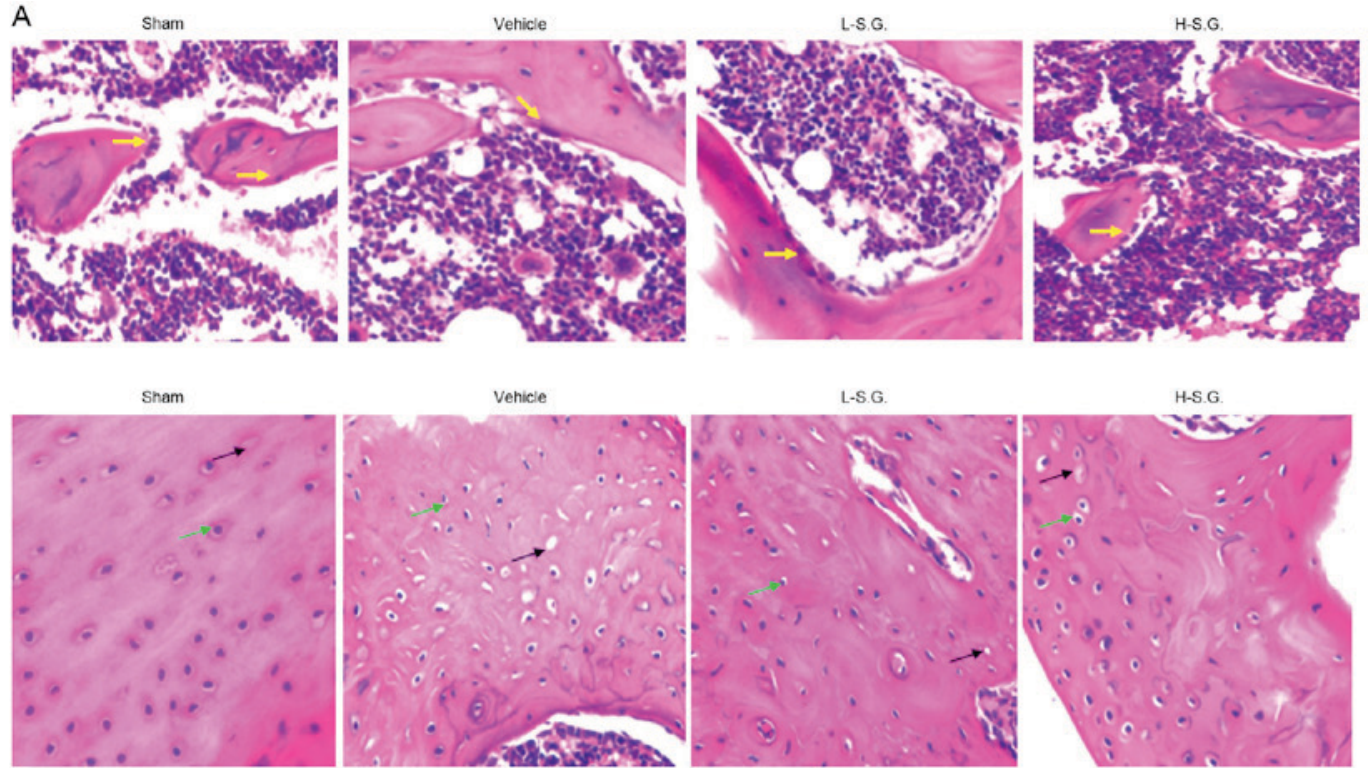

B
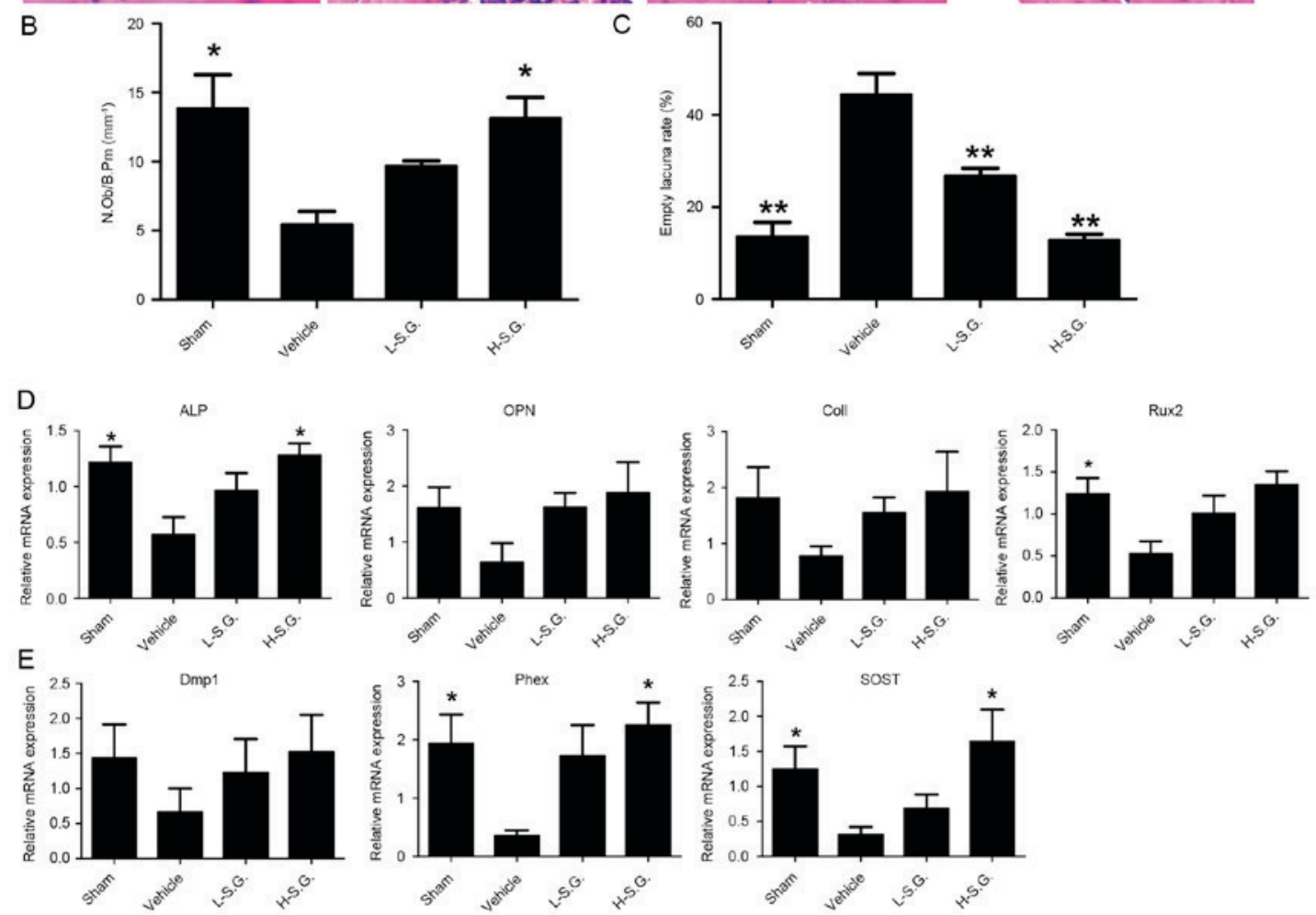

Figure 4. Sanguinarine promotes osteoblastic bone formation in vivo. Decalcified tissues were paraffin-embedded and sectioned for (A) hematoxylin and eosin staining, and the (B) N.Ob/B.Pm and (C) empty lacuna rate were calculated. (D) Expression of osteoblast-specific genes, including Runx2, ALP, OPN and Coll were analyzed using qPCR, and the results were normalized to the expression of $18 \mathrm{~S}$ and GAPDH. (E) Expression of osteocyte-specific genes, including Dmp1, Phex and SOST were analyzed by qPCR, and the results were normalized to the expression of $18 \mathrm{~S}$ and GAPDH. Magnification, $200 x$. ${ }^{*} \mathrm{P}<0.05$ and ${ }^{* *} \mathrm{P}<0.01$ vs. vehicle. N.Ob/B.Pm, number of osteoblasts/bone perimeter; Runx2, runt related transcription factor 2; ALP, alkaline phosphatase; OPN, osteopontin; ColI, Type I collagen; qPCR, quantitative polymerase chain reaction; Dmp1, dentin matrix acidic phosphoprotein 1; Phex, phosphate regulating endopeptidase homolog X-linked; SOST, sclerostin; 18S, 18S ribosomal RNA; L-S.G., low-dose sanguinarine group; H-S.G., high-dose sanguinarine group.

results indicate that sanguinarine may increase bone mass by protecting osteoblastic bone formation.

\section{Discussion}

Considering the wide use of sanguinarine as a traditional medicine and its inhibitory effect on osteoclasts in vitro, it was hypothesized that sanguinarine may have a role in protecting against osteoporosis in vivo. The present study revealed that sanguinarine protected mice from OVX-induced osteoporosis in a dose-dependent manner. This protective effect on osteoporosis may be due to suppressed osteoclast differentiation, as well as enhanced bone mineralization from enhanced osteoblast and osteocyte formation, which was supported by reduced osteoclast-specific gene expression, and increased osteoblast- and osteocyte-associated gene expression. Therefore, the results of the present study indicate a beneficial effect of sanguinarine on bone loss. 
Currently, the treatment for osteoporosis primarily focuses on bone remodeling performed by osteoclastic bone resorption and osteoblastic bone formation (17). The coupling process from bone resorption to formation is important for bone homeostasis (18). Suppressing bone resorption and enhancing bone formation are favorable options for preventing osteoporosis. For example, bisphosphonates increase the bone mineral density and reduce fractures by suppressing bone resorption, and also by exhibiting the side effect of delayed or absent fracture healing due to the inhibition of bone formation (19). Similarly, parathyroid hormone (PTH) increases bone formation while also stimulating resorption, which compromises its net anabolic effect on the bones (20). Therefore, a previous study proposed combining PTH and bisphosphonates for treating osteoporosis (21). Strontium ranelate has a dual effect, it stimulates osteoblast differentiation and inhibits osteoclast formation and resorption (22), and it has been used in clinical settings. Other drugs, including semaphorin 3A, N-oleoyl-L-serine and follicle stimulating hormone antibody may decouple bone remodeling; however, investigations are ongoing (23-25). Therefore, identification of a novel drug that promotes bone formation and reduces bone resorption simultaneously may be an appropriate direction for osteoporosis therapy.

There are several limitations to the present study. Although the results of the current study indicate that sanguinarine may affect osteoblasts and osteocytes, the mechanism of action of sanguinarine on osteoblasts and osteocytes requires further investigation. Furthermore, as the present study examined the effect of sanguinarine on bones, the potential side effects on mice were not investigated. The results of the present study demonstrated that the natural compound sanguinarine may prevent OVX-induced bone loss by inhibiting osteoclastic bone resorption and enhancing osteoblast bone formation in vivo, indicating that it is a potential option for preventing osteoporosis.

\section{Acknowledgements}

This study was supported by the National Nature Science Fund of China (grant nos. 81171739, 81101378, 81271971, 81271972 and 31270997), Natural Science Fund of Zhejiang Province (grant no. Y2110372), Funds of Science and Technology Department of Zhejiang Province (grant no. 2009C03014-1), and Zhejiang Provincial Program for the Cultivation of High-level Innovative Health Talents.

\section{References}

1. Sipos W, Pietschmann P, Rauner M, Kerschan-Schindl K and Patsch J: Pathophysiology of osteoporosis. Wien Med Wochenschr 159: 230-234, 2009

2. Weitzmann MN and Pacifici R: Estrogen deficiency and bone loss: An inflammatory tale. J Clin Invest 116: 1186-1194, 2006.

3. Rachner TD, Khosla S and Hof bauer LC: Osteoporosis: Now and the future. Lancet 377: 1276-1287, 2011.

4. Zhai YK, Pan YL, Niu YB, Li CR, Wu XL, Fan WT, Lu TL, Mei QB and Xian CJ: The importance of the prenyl group in the activities of osthole in enhancing bone formation and inhibiting bone resorption in vitro. Int J Endocrinol 2014: 921954, 2014.

5. Yogesh HS, Chandrashekhar VM, Katti HR, Ganapaty S, Raghavendra HL, Gowda GK and Goplakhrishna B: Anti-osteoporotic activity of aqueous-methanol extract of Berberis aristata in ovariectomized rats. J Ethnopharmacol 134 334-338, 2011.
6. Lee WS, Lee EG, Sung MS and Yoo WH: Kaempferol inhibits IL-1 $\beta$-stimulated, RANKL-mediated osteoclastogenesis via downregulation of MAPKs, c-Fos, and NFATc1. Inflammation 37: 1221-1230, 2014

7. Tyagi AM, Srivastava K, Singh AK, Kumar A, Changkija B, Pandey R, Lahiri S, Nagar GK, Yadav DK, Maurya R, et al: Formononetin reverses established osteopenia in adult ovariectomized rats. Menopause 19: 856-863, 2012.

8. Liu X, Qu X, Wu C, Zhai Z, Tian B, Li H, Ouyang Z, Xu X, Wang W, Fan Q, et al: The effect of enoxacin on osteoclastogenesis and reduction of titanium particle-induced osteolysis via suppression of JNK signaling pathway. Biomaterials 35: 5721-5730, 2014

9. Ouyang Z, Zhai Z, Li H, Liu X, Qu X, Li X, Fan Q, Tang T, Qin A and Dai K: Hypericin suppresses osteoclast formation and wear particle-induced osteolysis via modulating ERK signalling pathway. Biochem Pharmacol 90: 276-287, 2014.

10. Li H, Zhai Z, Liu G, Tang T, Lin Z, Zheng M, Qin A and Dai K: Sanguinarine inhibits osteoclast formation and bone resorption via suppressing RANKL-induced activation of NF- $\kappa B$ and ERK signaling pathways. Biochem Biophys Res Commun 430: 951-956, 2013.

11. Qu X, Zhai Z, Liu X, Li H, Ouyang Z, Wu C, Liu G, Fan Q, Tang T, Qin A and Dai K: Dioscin inhibits osteoclast differentiation and bone resorption though down-regulating the Akt signaling cascades. Biochem Biophys Res Commun 443: 658-665, 2014.

12. Mackraj I, Govender T and Gathiram P: Sanguinarine. Cardiovasc Ther 26: 75-83, 2008.

13. American Psychological Association: Guidelines for Ethical Conduct in the Care and Use of Nonhuman Animals in Research. American Psychological Association, Washington, DC, 2012. http://www.apa.org/science/leadership/care/guidelines.aspx.

14. Thompson DD, Simmons HA, Pirie CM and Ke HZ: FDA Guidelines and animal models for osteoporosis. Bone 17: 125S-133S, 1995.

15. Livak KJ and Schmittgen TD: Analysis of relative gene expression data using real-time quantitative PCR and the 2(-Delta Delta C(T)) Method. Methods 25: 402-408, 2001.

16. Bouxsein ML, Boyd SK, Christiansen BA, Guldberg RE, Jepsen KJ and Muller R: Guidelines for assessment of bone microstructure in rodents using micro-computed tomography. J Bone Miner Res 25: 1468-1486, 2010.

17. Das S and Crockett JC: Osteoporosis-a current view of pharmacological prevention and treatment. Drug Des Devel Ther 7: 435-448, 2013.

18. Buck DW III and Dumanian GA: Bone biology and physiology: Part I. The fundamentals. Plast Reconstr Surg 129: 1314-1320, 2012.

19. Odvina CV, Zerwekh JE, Rao DS, Maalouf N, Gottschalk FA and Pak CY: Severely suppressed bone turnover: A potential complication of alendronate therapy. J Clin Endocrinol Metab 90: 1294-1301, 2005.

20. Zaidi M and Iqbal J: Translational medicine: Double protection for weakened bones. Nature 485: 47-48, 2012.

21. Li YF, Zhou CC, Li JH, Luo E, Zhu SS, Feng G and Hu J: The effects of combined human parathyroid hormone (1-34) and zoledronic acid treatment on fracture healing in osteoporotic rats. Osteoporos Int 23: 1463-1474, 2012.

22. Bonnelye E, Chabadel A, Saltel F and Jurdic P: Dual effect of strontium ranelate: Stimulation of osteoblast differentiation and inhibition of osteoclast formation and resorption in vitro. Bone 42: 129-138, 2008.

23. Zhu LL, Blair H, Cao J, Yuen T, Latif R, Guo L, Tourkova IL, Li J, Davies TF, Sun L, et al: Blocking antibody to the $\beta$-subunit of FSH prevents bone loss by inhibiting bone resorption and stimulating bone synthesis. Proc Natl Acad Sci USA 109: 14574-14579, 2012

24. Smoum R, Bar A, Tan B, Milman G, Attar-Namdar M, Ofek O, Stuart JM, Bajayo A, Tam J, Kram V, et al: Oleoyl serine, an endogenous $\mathrm{N}$-acyl amide, modulates bone remodeling and mass. Proc Natl Acad Sci USA 107: 17710-17715, 2010.

25. Hayashi M, Nakashima $T$, Taniguchi M, Kodama $T$, Kumanogoh A and Takayanagi H: Osteoprotection by semaphorin 3A. Nature 485: 69-74, 2012. 